\title{
Complete Separation of Tyrosinated, Detyrosinated, and Nontyrosinatable Brain Tubulin Subpopulations Using Affinity Chromatography ${ }^{\dagger}$
}

\author{
Laurence Paturle, ${ }^{\ddagger}$ Juergen Wehland, ${ }^{\S}$ Robert L. Margolis, ${ }^{\prime}$ and Didier Job, ${ }^{* \ddagger}$ \\ Laboratoire BRCE, INSERM U 244, LBIO, DRF, Centre d'Etudes Nucléaires, BP 85X, 38041 Grenoble Cédex, France, Max \\ Planck Institute for Biophysical Chemistry, Postfach 2841, 3400 Goettingen, Federal Republic of Germany, and The Fred \\ Hutchinson Cancer Research Center, 1124 Columbia Street, Seattle, Washington 98104 \\ Received August 16, 1988; Revised Manuscript Received October 24, 1988
}

\begin{abstract}
The maximum achievable tyrosination level of neurotubulin, in vitro, is about $50 \%$. We have developed a method to obtain a complete separation of the tyrosinatable and nontyrosinatable species. We use an immunoaffinity column, with coupled YL 1/2 monoclonal antibody (anti-Tyr-tubulin) and rapid desalting methods. Both subpopulations can be obtained in a polymerizable, apparently native, form. We find that about $35 \%$ of the brain tubulin is truly nontyrosinatable, despite the fact that it is assembly competent. Using a polyclonal antibody directed against nontyrosinatable tubulin, we find that it recognizes a specific epitope on the $\alpha$-subunit of the dimer. The existence of an abundant tubulin subspecies, structurally different from tyrosinatable tubulin, should obviously be kept in mind in immunofluorescence studies of the distribution of nontyrosinated tubulin in brain tissues. Furthermore, we have extensively investigated the effect of tubulin tyrosination on microtubule dynamics. Despite the homogeneity of the populations under comparison, we find no significant effect of tyrosination on microtubule dynamics. Similarly, the stabilizing effects of microtubule associated proteins and of STOP protein were identical in both subpopulations. The drug taxol seems more efficient in stabilizing detyrosinated microtubules, but the difference is moderate. Taken together, these findings suggest that tubulin tyrosination does not effect microtubule stabilization, neither through modifications of the intrinsic tubulin properties nor through a differential binding of stabilizing proteins. Finally, the complete separation of two tubulin species (tyrosinated or detyrosinated) with similar kinetic properties, but immunologically different, should be of value in many kinetic studies of microtubule assembly.
\end{abstract}

$\mathbf{M}$ as determination of cell shape and motility, mitosis, or intracellular organelle transport (Dustin, 1984).

It is generally assumed that some microtubule differentiation should parallel this functional diversity (Schulze et al., 1987). Kinetic heterogeneities have been under particularly intensive investigation. Distinct subsets of drugs or temperature-resistant polymers have long been recognized in vitro and in vivo (Job et al., 1982). More recently, microtubules with different dynamic behavior have been shown to coexist in cells (Brê et al., 1987; Khawaja et al., 1988; Kreis, 1987; Schulze et al., 1987; Wehland \& Weber, 1987a).

Microtubule stability subclasses could be generated by the uneven binding of stabilizing proteins (Brê et al., 1987; Job et al., 1985), by covalent modifications of the tubulin dimers (Gard \& Kirschner, 1985; Wandosell et al., 1986), or by stability transitions due to some intrinsic property of tubulin (Mitchison \& Kirschner, 1984), or by interplays between these various mechanisms.

The reversible addition of a tyrosine residue to the carboxy terminus of $\alpha$-tubulin is the most remarkable covalent modification of this protein. It is both specific of the microtubule system and highly conserved during evolution (Thompson,

\footnotetext{
${ }^{\dagger}$ This work was supported in part by grants from the Ministère de la Recherche et de l'Enseignement Supêrieur and from the Mutuelle Génêrale de l'Education Nationale to D.J. This research was done during the tenure of a postdoctoral fellowship from the Ligue Nationale contre le Cancer to L.P.

$₫$ Centre d’Etudes Nuclēaires.

Max Planck Institute.

"The Fred Hutchinson Cancer Research Center
}

1982). It is therefore natural that the potential effect of tubulin tyrosination on microtubule dynamics has been a subject of intense scrutiny (Bré et al., 1987; Khawaja et al., 1988; Kreis, 1987; Thompson, 1982; Wehland \& Weber, 1987a).

In vivo, stable, less dynamic microtubules have been shown to be preferentially in the detyrosinated form (Brê et al., 1987; Khawaja et al., 1988; Kreis, 1987; Schulze et al., 1987; Wehland \& Weber, 1987a). There is evidence though that tyrosination is not causally related to stabilization (Khawaja et al., 1988; Schulze et al., 1987; Wehland \& Weber, 1987a).

In vitro, small differences have been observed between tyrosinated and detyrosinated microtubules (Kumar \& Flavin, 1982). However, the interpretation of these results is complicated by the nonhomogeneity of the microtubule populations under comparison: the maximal tyrosination level achieved in vitro is about $50 \%$. This raises two important questions. First, might sizable differences exist between fully tyrosinated or fully detyrosinated microtubules? Second, why is such a large amount of brain tubulin apparently nontyrosinatable? There are indications that it does not simply reflect enzymatic equilibrium (Flavin et al., 1982). Basic problems, though, remain unsolved: it is not known whether this species is denatured or not recognizable by the tubulin tyrosine ligase (Wehland \& Weber, 1987b) or if it represents a different tubulin form. Consequently, the extent of its contribution to the composition of "tyrosinated" or "detyrosinated" microtubules in previous in vitro studies is unclear.

The purpose of the present work has been to obtain a complete separation of tyrosinated and detyrosinated tubulin from nontyrosinatable tubulin. The potential effect of tubulin 
tyrosination on microtubule dynamics and on their interaction with various associated proteins could then be investigated by extensive comparisons of fully tyrosinated or detyrosinated polymers. It is also shown that nontyrosinatable tubulin is polymerizable and antigenetically different from the tyrosinatable species.

\section{Materials ANd Methods}

\section{Materials}

All chemicals, unless otherwise indicated, were purchased from Sigma Chemical Co. (St Louis, MO). $\left.{ }^{3} \mathrm{H}\right] \mathrm{GTP}(10$ $\mathrm{Ci} / \mathrm{mmol})$ was from Amersham. L- $\left[2,6{ }^{3} \mathrm{H}\right]$ Tyrosine $(50$ $\mathrm{Ci} / \mathrm{mmol}$ ) was from New England Nuclear (Boston, MA). Nucleotides, acetyl phosphate, and acetate kinase were from Boehringer Mannheim Biochemicals (Indianapolis, IN). Cyanogen bromide was from Flucka (Buchs, Switzerland). Sepharose 4B was from Pharmacia (Uppsala, Sweden). GF/C glass fiber filters and phosphocellulose P11 were from Whatman Inc. (Clifton, NJ). Bio-Gel P-6 for gel filtrations was from Bio-Rad Laboratories (Richmond, CA). Nitrocellulose membrane filters (BA83, $0.2 \mu \mathrm{M}$ ) were from Schleicher \& Schuell (Dassel, FRG). Carboxypeptidase A was from Serva (New York). The buffer used for protein purification was $100 \mathrm{mM}$ MES [2-( $N$-morpholino)ethanesulfonic acid], $1 \mathrm{mM} \mathrm{MgCl}$, and $1 \mathrm{mM}$ EGTA [ethylene glycol bis ( $\beta$-aminoethyl ether)- $N, N, N^{\prime}, N^{\prime}$-tetraacetic acid], pH 6.75 (designated MME). PME buffer (pH 6.65) has the same constitution as MME, except that PIPES [piperazine$N, N^{\prime}$-bis(2-ethanesulfonic acid)] was used in place of MES. Protein solutions were concentrated by ultrafiltration through Centricon 30 (Amicon, Danvers, MA).

Taxol was a generous gift of Dr. M. Wright. The antibody conjugated to alkaline phosphatase and the color development substrates [NBT (nitro blue tetrazolium) and BCIP (5bromo-4-chloro-3-indolyl phosphate)] were from Promega Biotec (Madison, WI).

The monoclonal YL $1 / 2$ antibody was a generous gift of Dr. J. V. Kilmartin. This antibody specifically recognizes the carboxy-terminal residue of the $\alpha$-subunit of tyrosinated tubulin (Wehland et al., 1983).

\section{Methods}

Microtubule Protein Isolation. Cold labile microtubule protein from beef brain (refered to as MAP microtubule protein) was isolated by three cycles of assembly and disassembly in MME buffer, according to published procedures (Job et al., 1982).

Pure tubulin was isolated from MAP microtubule protein by phosphocellulose column chromatography in MME buffer, from which pure tubulin elutes in the flow-through fraction (Weingarten et al., 1975) and was further concentrated to 5 $\mathrm{mg} / \mathrm{mL}$ by ultrafiltration.

Purification of Tubulin Tyrosine Ligase. Tubulin tyrosine ligase was isolated from porcine brain by immunoaffinity chromatography as described in detail elsewhere (Wehland et al., 1986), using a mouse monoclonal antibody specific for the ligase (Schroder et al., 1985). The enzyme was eluted from the immobilized antibody with $3 \mathrm{M} \mathrm{MgCl}_{2}$ in stabilization buffer [25 mM K+-MES, pH 6.8, $100 \mathrm{mM} \mathrm{KCl,} 2 \mathrm{mM}$ $\mathrm{MgCl}_{2}, 1 \mathrm{mM}$ EGTA, $0.5 \mathrm{mM}$ DTT, $20 \%$ glycerol $\left.(\mathrm{v} / \mathrm{v})\right]$, dialyzed against the stabilization buffer, and stored at $-70^{\circ} \mathrm{C}$.

Protein Sample Desalting. Rapid desalting was performed by a centrifugation-filtration method on Bio-Gel P-6 (Penefsky et al., 1977).

Immunoaffinity Column. The rat monoclonal antibody YL $1 / 2$ was coupled to cyanogen bromide activated Sepharose 4B according to Cuatrecasas (1970).

The use of freshly activated gels was found to be a prerequisite to obtain reproducible results. Affinity chromatography was always performed in PME buffer. The affinity column contained $5 \mathrm{mg}$ of YL $1 / 2$ antibody per milliliter of activated gel.

Tubulin Tyrosination and Tubulin Tyrosine Ligase Assay. Tyrosination was performed according to Wehland and Weber (1987b) with minor modifications. The final pure tubulin concentration in the assay was $4.5 \mathrm{mg} / \mathrm{mL}$. We used $5 \mathrm{mM}$ ATP and $25 \mathrm{mg} \mathrm{MgCl}_{2}$ (final concentration) for maximal tyrosination.

When needed, tyrosine incorporation was monitored with $\left[{ }^{3} \mathrm{H}\right]$ tyrosine $(2 \mu \mathrm{Ci} / 50 \mu \mathrm{L})$. The amount of tyrosine in tubulin was further assayed according to Sandoval and Cuatrecasas (1976).

Other Assays. Turbidimetric assay of microtubule assembly was as in Pirollet et al. (1987). The filter assay of microtubule assembly and the microtubule-associated protein (MAP) assay have been described in previous reports (Job et al., 1985). Briefly, for MAP assay pure tubulin microtubules are assembled in the presence of $10^{-4} \mathrm{M} \mathrm{GTP}, 10 \mathrm{mM}$ acetyl phosphate, $0.5 \mu \mathrm{g} / \mathrm{mL}$ acetate kinase, and $2 \mu \mathrm{Ci}$ of $\left[{ }^{3} \mathrm{H}\right] \mathrm{GTP} / \mathrm{mL}$. After full assembly they are diluted 25 times in $50 \%$ sucrose containing MME buffer and either immediately cross-linked with $0.75 \%$ glutaraldehyde (controls) or incubated with MAPs. The mixture is further diluted to $30 \%$ sucrose by addition of MME buffer. After $10 \mathrm{~min}$, the surviving polymers are cross-linked. The amount of labeled nucleotide in polymers is then determined by filtration of the solution on GF/C filters as previously described (Job et al, 1985). The proportion of surviving microtubules is a measurement of the MAP content.

A full description of the STOP assay will be presented elsewhere. Briefly, STOP was partially purified by chromatography of beef brain crude extracts on a heparin ultrogel column.

The STOP assay is similar to the MAP assay except that assembled microtubules are diluted in $40 \%$ sucrose containing MME buffer, incubated with various amounts of STOP-containing fractions, and further left on ice for $40 \mathrm{~min}$. The amount of surviving polymers is a measure of their STOP content.

Western Blots. They were processed according to the methods of Towbin et al. (1979), using a secondary antibody containing alkaline phosphatase as a probe.

Polyclonal Antibody to Nontyrosinatable Tubulin. The antibody was raised in a rabbit according to an injection schedule with $600 \mu \mathrm{g}$ of nontyrosinatable tubulin. IgG were purified according to McKinney and Parkinson (1987). To eliminate the immunoglobulins which recognized epitopes shared by the various tubulin subspecies, $\operatorname{IgG}(50 \mu \mathrm{g} / \mathrm{mL}, 10$ $\mathrm{mL}$ ) were incubated sequentially with four nitrocellulose sheets onto which tyrosinatable, detyrosinated tubulin had previously been blotted (Towbin et al., 1979) (180 $\mu \mathrm{g}$ of tyrosinatable tubulin per blot). The final supernatant contains essentially the IgGs specific to nontyrosinatable tubulin.

\section{RESUltS}

Separation of Tyrosinated and Nontyrosinatable Tubulin. As described under Materials and Methods, we used an affinity chromatographic procedure to isolate tyrosinated from nontyrosinatable tubulin. Maximally tyrosinated pure tubulin containing $\left[{ }^{3} \mathrm{H}\right]$ tyrosine is loaded onto a column of immobilized YL 1/2 antibody (anti-Tyr-tubulin). Nontyrosinated tubulin is not retained on the column. The tyrosinated species is eluted in a salt-containing buffer. The relative proportion of each 


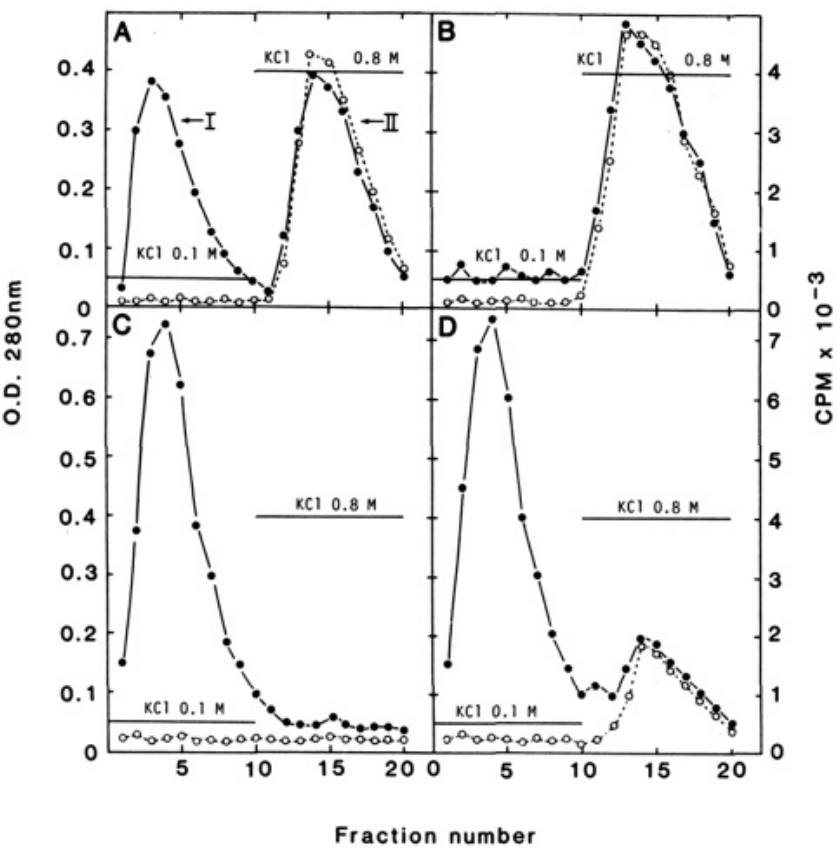

FIGURE 1: Affinity chromatography of tyrosinated and nontyrosinated tubulin. (A) Pure tubulin $(4.5 \mathrm{mg} / \mathrm{mL})$ in MME buffer was maximally tyrosinated in the presence of $\left[{ }^{3} \mathrm{H}\right]$ tyrosine, exchanged in PME buffer, and loaded onto a YL 1/2 immunoaffinity column at a concentration of $2 \mathrm{mg} / \mathrm{mL}$ of gel. The column was further eluted with 5 volumes of PME buffer containing $0.1 \mathrm{M} \mathrm{KCl}$. A stepwise elution was then performed with the same volume of PME buffer containing $0.8 \mathrm{M} \mathrm{KCl}$. Fractions (half a column volume) were eluted directly onto Bio-Gel P-6 equilibrated in PME buffer for immediate desalting (see Materials and Methods). They were then assayed for protein content by $\mathrm{OD}_{280}$ measurement $(\bullet)$ and for $\left[{ }^{3} \mathrm{H}\right]$ tyrosine incorporation by radioactivity counting of a $40-\mu \mathrm{L}$ aliquot (O). (B) Fractions from peak II (panel A) were pooled, concentrated $(5 \mathrm{mg} / \mathrm{mL}$ protein concentration), and rechromatographed as in (A): (๑) $\mathrm{OD}_{280} ;(\mathrm{O})$ $\mathrm{cpm}$. (C) Pure tubulin $(4.5 \mathrm{mg} / \mathrm{mL})$ was incubated for $10 \mathrm{~min}$ at $30^{\circ} \mathrm{C}$ with carboxypeptidase $\mathrm{A}(2 \mu \mathrm{g} / \mathrm{mL})$ and further chromatographed as in (A): (๑) $\mathrm{OD}_{280} ;(0) \mathrm{cpm}$. (D) Fractions from peak I (panel A) were pooled and concentrated to a $5 \mathrm{mg} / \mathrm{mL}$ protein concentration. They were then maximally tyrosinated and chromatographed as in (A): (๑) $\mathrm{OD}_{280} ;(0) \mathrm{cpm}$.

species is around $50 \%$ as usually observed with other procedures (Figure 1A).

In the general case, this type of procedure yields denatured tubulin. Two factors were found to be of importance to obtain polymerizable protein: the antibody coupling procedure and the immediate desalting of the eluted fractions, as described under Materials and Methods and in the figure legends.

To demonstrate the homogeneity of the two peaks, we performed various rechromatography experiments: Figure 1B,C shows that fully tyrosinated or detyrosinated tubulin elutes in two distinct peaks corresponding respectively to peaks II and I of Figure 1A. When nontyrosinated tubulin is subjected to a second cycle of tyrosination and rechromatographed, only a few percent incorporate $\left[{ }^{3} \mathrm{H}\right]$ tyrosine and bind to the column (Figure 1D). This result shows that the initial almost even separation of tubulin between the tyrosinated and nontyrosinated species does not simply reflect enzymatic equilibria.

A third cycle of tyrosination of the tubulin contained in the major peak shown in Figure 1D results in nondetectable incorporation of tyrosine as judged by both radioactivity incorporation and column chromatography (not shown).

The eluted protein has been further probed for homogeneity with an immunoblotting technique (Figure 2A). Three species are under comparison: tyrosinated tubulin (from peak II, Figure 1A), detyrosinated tubulin obtained by incubation of

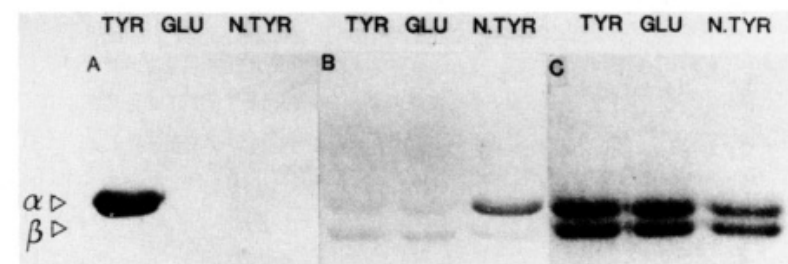

FIGURE 2: Western blot analysis of the different tubulin species, using YL 1/2 (anti-Tyr-tubulin) (A) or a polyclonal anti-nontyrosinatable tubulin antibody (B) as probe. (A) Tyrosinated tubulin (TYR) and nontyrosinatable tubulin (N.TYR) were directly obtained from column fractions corresponding to peak II, Figure 1A, and to the major peak, Figure 1D, respectively. Detyrosinated (GLU) tubulin was obtained by incubation of the tyrosinated species with carboxypeptidase $A$ as described in Figure 1C. Equal quantities $(5 \mu \mathrm{g})$ of each tubulin species were run into 8\% SDS-polyacrylamide gels, transferred onto nitrocellulose sheets, and immunoblotted with YL 1/2, as described under Materials and Methods. (B) Same as above using a polyclonal antibody against nontyrosinatable tubulin as a probe. (C) Corresponding SDS-polyacrylamide gel of materials analyzed in (A).

the same species with carboxypeptidase A, and nontyrosinatable tubulin, contained in the major peak shown in Figure 1D. The only species recognized by the YL $1 / 2$ monoclonal antibody is, as expected, the tubulin from peak II. Figure 2B shows a comparison of the same subspecies as in Figure 2A with the difference that the primary antibody used is a specific polyclonal antibody directed against nontyrosinatable tubulin and repeatedly absorbed on tyrosinatable tubulin (see Materials and Methods). This antibody still shows some "background" activity directed against $\alpha$ - and $\beta$-subunits from the three isotypes but also exhibits a clear specific reaction with the $\alpha$-subunit of the nontyrosinatable tubulin. Thus, the result reveals a specific epitope onto the $\alpha$-subunit of this tubulin subspecies. Since tubulin acetylation and detyrosination seem to be correlated in vivo (Bulinski et al., 1988), we have investigated whether acetylation could be responsible for the specific immunological reactivity of the nontyrosinatable $\alpha$-subunit. Tyrosinatable tubulin was chemically acetylated as in Piperno and Fuller (1985), and the experiment shown in Figure 1B was repeated, introducing this modified protein in the comparison. These results (not shown) showed that acetylated tyrosinatable tubulin was not recognized by the antibody, suggesting that this covalent modification is not responsible for the specific reactivity of the nontyrosinatable tubulin $\alpha$-subunit.

Finally, Figure $2 \mathrm{C}$ is a control showing that comparable amounts of the various tubuline isotypes have been blotted.

Figure 3 shows that the three populations are made of polymerizable tubulin. Furthermore, there is no obvious difference between the assembly curves. This is important because it shows that the nontyrosinatable tubulin is not simply denatured and that, in previous studies, this tubulin species was incorporated into microtubules together with the detyrosinated or tyrosinated dimers.

Kinetic Properties of Tyrosinated and Enzymatically Detyrosinated Tubulin. The principal results of a series of extensive comparisons of the tyrosinated and detyrosinated species are shown in Figures 4-6. They show that the critical concentrations are the same for the two species (Figure 4). This experiment has been repeated in different buffers, especially phosphate glutamate buffer which might be more "physiological" than Pipes buffers, with the same result (not shown).

Similarly, the rate of subunit exchange at equilibrium is identical in all tested conditions (Figure 5). Finally, we have recently described conditions in which tubulin polymerizes 


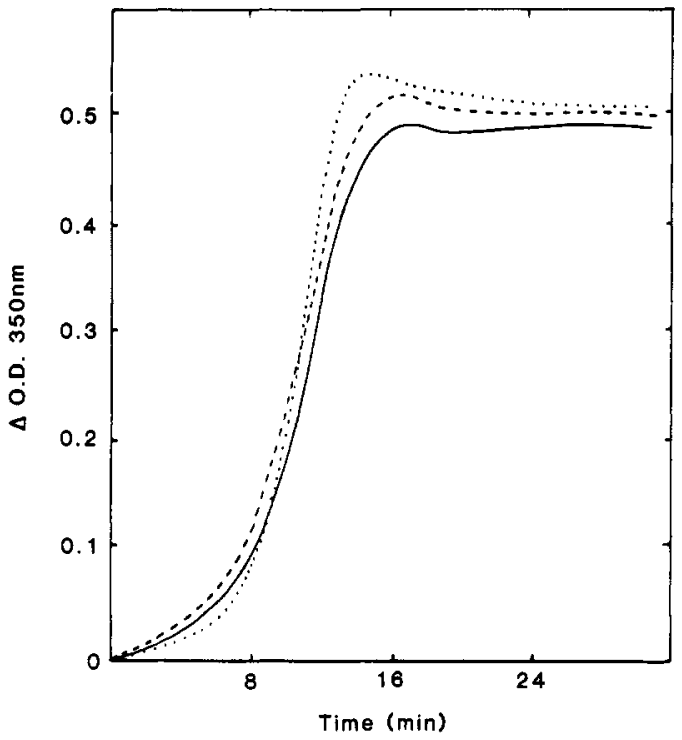

FIGURE 3: Polymerization of the purified tubulin subpopulations. Tyrosinated or nontyrosinatable tubulin species were obtained from column fractions as described in Figure 2. They were exchanged in PME buffer and further concentrated to $4.5 \mathrm{mg} / \mathrm{mL}$. Half of the sample containing tyrosinated tubulin was treated with carboxypeptidase $\mathbf{A}$ to generate detyrosinated tubulin, as described in Figure 1C. The reaction was terminated by the addition of dithiothreitol to a final concentration of $20 \mathrm{mM}$ (Kumar \& Flavin, 1982). The two other samples were adjusted to the same dithiotreitol concentrations. All samples were adjusted to $10 \mathrm{mM} \mathrm{MgCl}$ and $1 \mathrm{mM} \mathrm{GTP}$, placed in microcuvettes, and assembled at $37^{\circ} \mathrm{C}$ in a spectrophotometer. Final protein concentration was $4 \mathrm{mg} / \mathrm{mL}$. (-) Tyrosinated tubulin; (-) detyrosinated tubulin; (...) nontyrosinatable tubulin.

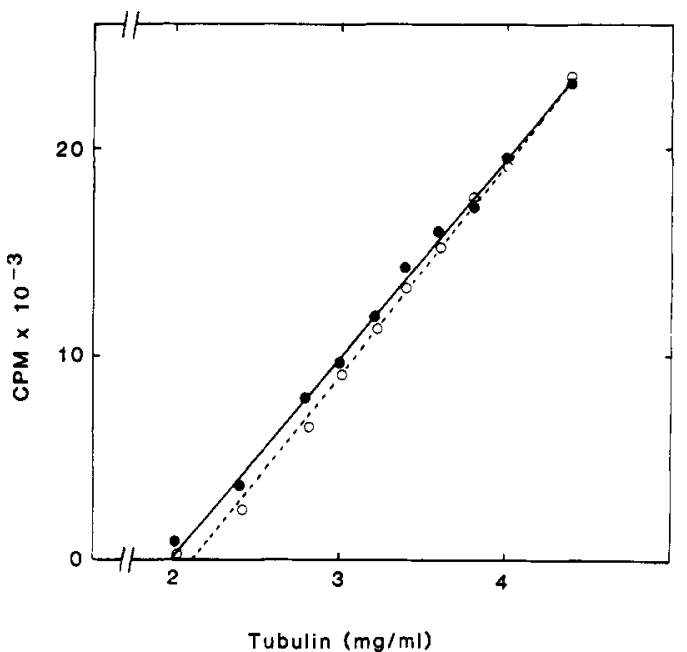

FIGURE 4: Effect of tubulin tyrosination on critical concentrations for microtubule assembly. Tyrosinated or detyrosinated tubulin species were prepared as described in Figure 3. Aliquots $(50 \mu \mathrm{L})$ containing variable amounts of tubulin were assembled for $40 \mathrm{~min}$ at $37^{\circ} \mathrm{C}$ in PEM buffer containing $10 \mathrm{mM} \mathrm{MgCl}_{2}$, and the reaction mixture was used for filter assay of microtubule assembly (see Materials and Methods). Final tubulin concentrations are indicated in the abscissa. Each point is the mean value of two replicates. (-) Tyrosinated tubulin; $(0)$ detyrosinated tubulin.

following an oscillatory pathway (Pirollet et al., 1987). Under these conditions microtubule oscillations are almost superimposable in the two populations (Figure 6).

They were also indistinguishable with regard to the effects of $\mathrm{Ca}^{2+}$ (Figure 7), a divalent ion which is supposed to mainly interact with the C-terminus of the $\alpha$-tubulin.

Finally, we find a moderate but reproducible effect of tubulin tyrosination on taxol-induced microtubule stabilization (Figure 8). Thus, detyrosinated polymers are more resistant

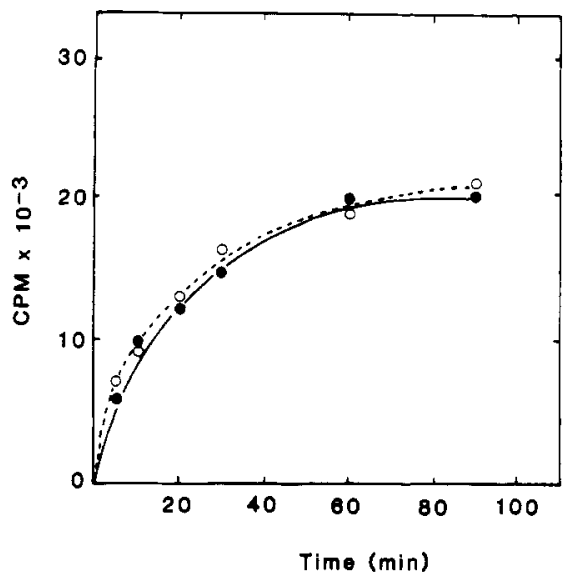

FIGURE 5: Effect of tyrosination on steady-state tubulin incorporation into microtubule. Tyrosinated or detyrosinated tubulin species were prepared as described in Figure 3. The two samples containing 4.5 $\mathrm{mg} / \mathrm{mL}$ (final concentrations) were assembled for $40 \mathrm{~min}$ as in Figure 4 , with the exception that $\left[{ }^{3} \mathrm{H}\right] \mathrm{GTP}$ was omitted in the reaction mixture. The labeled nucleotide was then added at apparent steady state, and radioactivity incorporation into microtubules was monitored on $20-\mu \mathrm{L}$ aliquots at the indicated time points. The filter assay for radioactivity determination is described under Materials and Methods. (๑) tyrosinated tubulin; (O) detyrosinated tubulin. Each measurement is the mean value of two replicates.

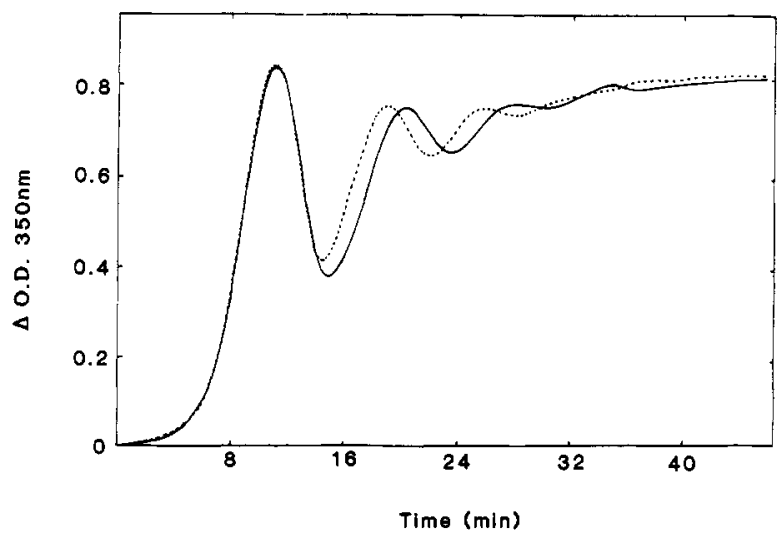

FIGURE 6: Oscillatory assembly of microtubules: effect of tubulin tyrosination. Tyrosinated or detyrosinated tubulin species were prepared as described in Figure 3 and further concentrated by ultrafiltration. The two samples were adjusted at final concentrations of $6.2 \mathrm{mg} / \mathrm{mL}$ tubulin, $10 \mathrm{mM} \mathrm{MgCl}, 10 \mathrm{mM}$ acetyl phosphate, and $0.5 \mu \mathrm{g} / \mathrm{mL}$ acetate kinase, placed in microcuvettes, and assembled at $37^{\circ} \mathrm{C}$ in a spectrophotometer. (-) Tyrosinated tubulin; (...) detyrosinated tubulin.

than the tyrosinated species to cold-induced depolymerization in the presence of the drug.

Binding of Associated Proteins to Tyrosinated or Detyrosinated Microtubules. We have previously developed quantitative assays of the effect of MAPs (HMW MAPs or $\tau$ proteins) on microtubule stability (Job et al., 1985). A similar assay, to be fully described elsewhere, has been used to investigate the effect of STOPs (Margolis et al., 1986). STOP is the most potent microtubule stabilizing protein (Job et al., 1987; Margolis et al., 1986), and we have recently shown that it has a widespread distribution among mammalian tissues (unpublished results).

Once more the behavior of tyrosinated or nontyrosinated polymers was found to be similar: MAPs (Figure 9A) or STOPs (Figure 9B) stabilized both polymer populations with essentially the same concentration dependency. It can be noted that in the results shown MAPs are added as MAP microtubule protein, as in Job et al. (1985). The same results can be obtained with purified MAPs or $\tau$ protein. 


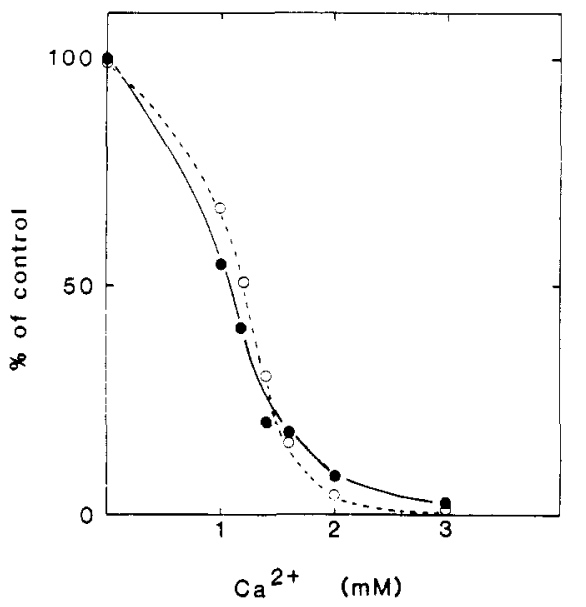

FIGURE 7: Effect of tyrosination on $\mathrm{Ca}^{2+}$-induced disassembly of microtubules. Tyrosinated or detyrosinated tubulin species were prepared as described in Figure 3. Aliquots $(20 \mu \mathrm{L})$ containing 4 $\mathrm{mg} / \mathrm{mL}$ tubulin, $10 \mathrm{mM} \mathrm{MgCl}$, and the reaction mixture used for filter assay of microtubule assembly (see Materials and Methods) were incubated for $40 \mathrm{~min}$ at $37^{\circ} \mathrm{C}$. $\mathrm{Ca}^{2+}(2 \mu \mathrm{L})$ was then added to the indicated final concentrations, and the samples were incubated for an additional $10 \mathrm{~min}$. They were then filter assayed for residual assembly levels. Results are expressed as percent of control, measured in the absence of $\mathrm{Ca}^{2+}$ addition. (๑) Tyrosinated tubulin; (O) detyrosinated tubulin.

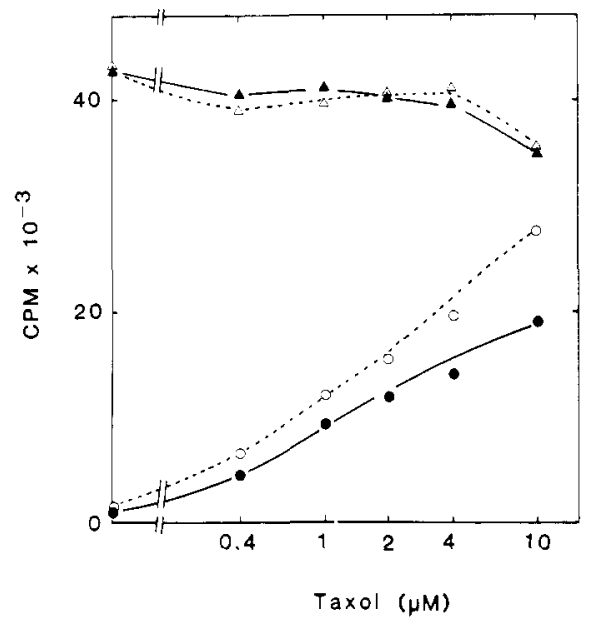

FIGURE 8: Effect of tubulin tyrosination on taxol-induced microtubule stabilization. Tyrosinated or detyrosinated tubulin species were prepared as described in Figure 3. It was aliquoted $(40 \mu \mathrm{L})$ and incubated for $30 \mathrm{~min}$ at $30^{\circ} \mathrm{C}$ at a final protein concentration of 1.5 $\mathrm{mg} / \mathrm{mL}$, in PME buffer containing $20 \%$ glycerol, $15 \mathrm{mM} \mathrm{MgCl}_{2}$, and the reaction mixture used for filter assay of microtubule assembly (see Materials and Methods). Taxol $(2 \mu \mathrm{L})$ was then added in DMSO, at the proper concentration to obtain the indicated final concentrations. The tubes were incubated for an additional $15 \mathrm{~min}$ and either im. mediately processed for filter assay (half of the tubes) or placed in ice for $30 \mathrm{~min}$ prior to further processing. (А) Assembly level of tyrosinated tubulin in the presence of taxol, at $30^{\circ} \mathrm{C} ;(\Delta)$ same as above for detyrosinated tubulin; (๑) residual assembly level of tyrosinated tubulin, in the presence of taxol, after $30 \mathrm{~min}$ on ice. (O) Same as above for detyrosinated tubulin. Note the use of a glycerol-containing buffer and of relatively low protein concentrations in this particular experiment. Under the conditions used in other experiments (PME buffer containin $10 \mathrm{mM} \mathrm{MgCl}$, higher protein concentration), we observed a rapid apparent denaturation of tubulin.

Other Comparisons of the Tyrosinated and Detyrosinated Tubulin Species. Since the above-reported experiments failed to indicate any significant effect of tubulin tyrosination on the polymerization process, we decided to investigate its possible action on microtubule lateral interactions. Repeated viscosimetric experiments using a falling ball apparatus revealed no differences. However, we found this kind of measurement
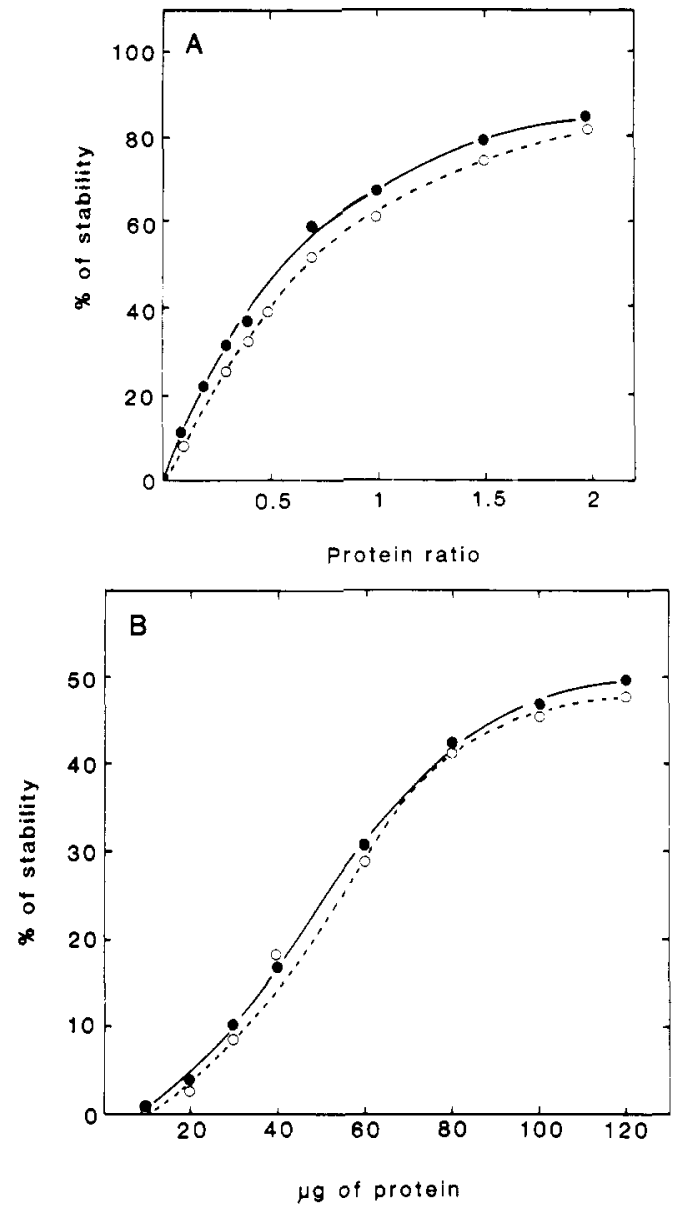

FIGURE 9: Effect of tyrosination on MAP- and STOP-induced stabilization of microtubules. Tyrosinated and detyrosinated tubulin species were prepared as described in Figure 3. Assembly and assay conditions were as described under Materials and Methods. Doseeffect curves for microtubule stabilization are shown for MAPs (A) and STOPs (B). MAPs were added as unpolymerized microtubule protein [see Job et al. (1985)]. STOPs were partially purified from beef brain crude extracts as described under Materials and Methods. (•) Tyrosinated tubulin; (O) detyrosinated tubulin

to be poorly reproducible when dealing with pure tubulin microtubules. Similarly, neutron scattering experiments failed to show any modification of the spectra that would indicate different spacial arrangements of microtubules (J. Tabony, L. Paturle, D. Job, unpublished results).

Finally, we have used previously described techniques (Brown \& Berlin, 1985) to measure the specific volume of tyrosinated and detyrosinated polymers. The observed values were $6.21 \mu \mathrm{L} / \mathrm{mg}$ of protein and $6.54 \mu \mathrm{L} / \mathrm{mg}$ of protein for tyrosinated and detyrosinated microtubules, respectively, not significantly different.

Nontyrosinatable Tubulin. This isotype could not be included in the comparative studies shown above for several reasons.

First, there is no firm evidence as yet that it represents a homogeneous population. Several different modifications of the $\alpha$-subunit might generate nontyrosinatable tubulin.

More importantly, we have learned during the initial steps of the present work that, in order to be unbiased and reproducible, comparisons of tubulin samples should use extensively purified protein preparations obtained with exactly the same preparative protocol. These criteria apply quite ideally to tyrosinated and detyrosinated subspecies but not to nontyrosinatable tubulin.

The solution to these problems of homogeneity, purity, and preparative protocol will have to rely on the development of 


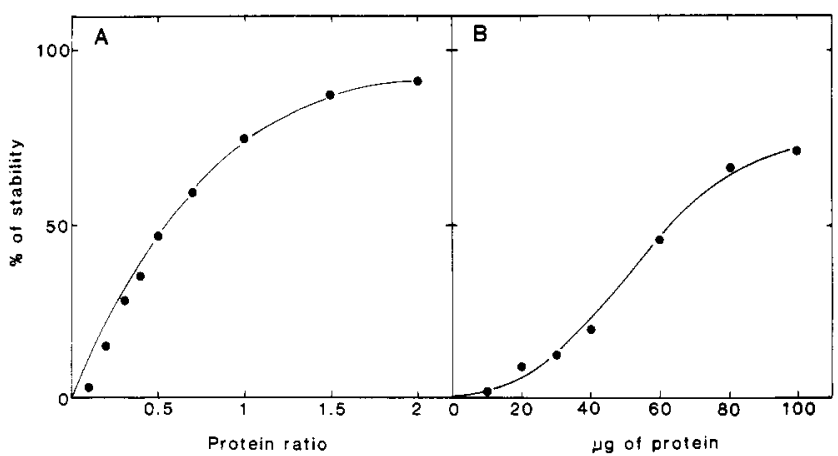

FIGURE 10: Stabilizing effect of MAPs and STOPs on nontyrosinatable tubulin. Nontyrosinatable tubulin was prepared as in Figure 3. Other conditions were as in Figure 9 with the exception that nontyrosinatable tubulin was used instead of the tyrosinatable isotypes.

monoclonal antibodies directed against nontyrosinatable tubulin.

Meanwhile, in separate experiments, we have performed a preliminary survey of the kinetic properties of nontyrosinatable tubulin. We find nothing atypical about this subspecies which exhibits subunit exchange at equilibrium, $\mathrm{Ca}^{2+}$ sensitivity, and so on.

Similarly, it shows the usual sensitivity to the stabilizing effect of MAPs and STOPs (Figure 10).

\section{Discussion}

This paper confirms previous studies which failed to detect any large effect of tubulin tyrosination on microtubule dynamics and in which the existence of nontyrosinatable tubulin was suspected (Thompson, 1982; Flavin et al., 1982). Obviously, these conclusions are now established on a much firmer basis because of the homogeneity and/or of the demonstrated polymerizable state of the tubulin subpopulations under investigation, and also because the immunological differences that we find between tyrosinatable and nontyrosinatable tubulin.

We do find some effect of tyrosination on the taxol-induced stabilization of microtubules: this drug seems more efficient when the tubulin is in the detyrosinated form. However, this effect is moderate. In a previous report, taxol was found to have the reverse effect on tyrosinated and nontyrosinated tubulin polymers (Kumar \& Flavin, 1982). The reasons for this discrepancy are not obvious. The interpretation is complicated by the denaturing effect of the drug on pure tubulin which is marked for drug concentrations stoichiometric to tubulin. This denaturing effect is much more severe when affinity-purified tubulin is used, as in the present study, than when phosphocellulose-purified tubulin is used. Small amounts of contaminating microtubule proteins in ion exchange purified preparations might well account for this difference.

By all other criteria, tyrosinated and detyrosinated tubulin behave similarly. The complete separation of these two tubulin species achieved in the present work eliminates the possibility that the lack of detection of sizable differences can be ascribed to the heterogeneity of the preparations under comparison, as in previous work. Furthermore, we have tested additional kinetic parameters. For example, we believe that the observation of almost superimposable assembly curves under oscillation conditions (Pirollet et al., 1987) is of special interest because this complex phenomenon depends on a set of numerous kinetic constants.

The extrapolation of in vitro results to the in vivo situation is always questionable. Nevertheless, these results make a direct participation of tubulin tyrosination in the generation of microtubule stability subclasses in cell highly improbable.
Tubulin tyrosination could act by modifying microtubule affinity for stabilizing proteins (Schulze et al., 1987). We find no indication for such a mechanism, even using the quantitative MAP assay and introducing a new effector, STOP protein, in the comparisons. However, the endogenous MAPs of the cycling nonneuronal cells used for cytological experiments have not as yet been tested for differential binding to tyrosinated or detyrosinated microtubules.

As recently suggested (Khawaja et al., 1988; Schulze et al., 1987; Wehland \& Weber, 1987b), the most likely explanation for the observed association between detyrosination and $\mathrm{mi}$ crotubule stability presently is that stable polymers are exposed longer to endogenous carboxypeptidases.

The initial establishment of microtubule stability subclasses has been ascribed to polymer stabilization by capping proteins in selected regions of the cell (Kirschner \& Mitchison, 1986) or to the uneven binding of subsaturating microtubule-associated proteins (Job et al., 1985). The two mechanisms should be distinguishable, because in the latter case polymer stability should reflect the initial order of growth (Job et al., 1985). It should thus be most interesting to known if tyrosination differences are generated on a "first to grow" basis (Brê et al., 1987).

During the process of purification of tyrosinated and detyrosinated tubulin, we have demonstrated that about $35 \%$ of brain tubulin is not tyrosinatable, despite the fact that it is not obviously denatured. Furthermore, using a specific polyclonal antibody, we find that the $\alpha$-subunit of nontyrosinatable tubulin is structurally different from the corresponding subunits of the tyrosinatable species. This observation is of potential importance: nontyrosinated tubulin has been detected in various parts of the nervous system (Cambray \& Burgoyne, 1987). One clearly needs to know whether it is generated by the classical tyrosination-detyrosination cycle of $\alpha$-tubulin or if it reflects the tissue distribution of nontyrosinatable tubulin. It would also be important to know if this latter species is brain specific or if it can also be found in other tissue. It is obvious that the molecular basis of this remarkable tubulin resistance to tyrosination will have to be elucidated. The nontyrosinatable species could be generated by several nonmutually exclusive mechanisms: covalent modifications of the tubulin dimer and/or differences in the primary structure of the protein.

The second possibility is particularly attractive in view of the well-documented sequence variability of the tubulin $\alpha$ subunit in mammalians [for example, see Villasante et al. (1986), Cleveland (1987), Gu et al. (1988), and Lewis and Cowan (1988)].

Thus five different tubulin isotypes have been described in mammalians (Villasante et al., 1986), called $\mathrm{M} \alpha 1, \mathrm{M} \alpha 2$, $\mathrm{M} \alpha 3 / 7, \mathrm{M} \alpha 4$, and $\mathrm{M} \alpha 6$ in mouse. $\mathrm{M} \alpha 3 / 7$ is expressed exclusively in testis, $\mathrm{M} \alpha 6$ is expressed in many tissues but at very low levels ( $\mathrm{Gu}$ et al., 1988). M $\alpha 4$ is found in brain and is unique among mammalian $\alpha$-tubulins because it lacks an encoded carboxy-terminal tyrosine residue. Despite this remarkable feature, it has recently been shown to go, in vivo, through the normal cycle of tyrosination and detyrosination (Gu et al., 1988).

Finally, $\mathrm{M} \alpha 1$ and $\mathrm{M} \alpha 2$ are widely expressed and particularly abundant in cultured cells. Most of the work done on the turnover of the $\alpha$-tubulin carboxy-terminal tyrosine has dealt with these isotypes ( $\mathrm{Gu}$ et al., 1988) which are thus known to normally be substrates of the tyrosine ligase.

Taken together, these results do not suggest any clear relationship between tyrosination and the sequence variations of the tubulin $\alpha$-subunit. 
However, the situation might be different in vitro. The availability of nontyrosinatable tubulin away from the other subspecies should be of value in order to test this hypothesis.

The physiological significance of tubulin tyrosination remains unknown. Further in vitro or in vivo studies assaying different microtubule functions are clearly needed. For example, it would be of particular interest to find out if tyrosination influences organelle transport along microtubules, a phenomenon which has recently been shown to be independent of their kinetic properties (Seitz-Tutter et al., 1988). The possibility to compare tubulin subsets that are homogeneous with respect to tyrosination should be of value in the process.

The complete separation of tyrosinated and detyrosinated tubulin subpopulations by affinity chromatography should also be most useful for further in vitro studies. It yields preparations made of pure tubulin, exhibiting the same kinetic behavior, which are nevertheless immunologically easy to identify.

\section{ACKNOWLEDGMENTS}

We thank D. Divers for excellent technical assistance during this work.

Registry No. Taxol, 33069-62-4.

\section{REFERENCES}

Brē, M. H., Kreis, T. E., \& Karsenti, E. (1987) J. Cell Biol. 105, 1283-1296.

Brown, P. A., \& Berlin, R. D. (1985) J. Cell Biol. 101, 1492-1500.

Bulinski, J. C., Richards, J. E., \& Piperno, G. (1988) J. Cell Biol. 106, 1213-1220.

Cambray, M. A., \& Burgoyne, R. D. (1987) J. Cell Biol. 104, $1569-1574$

Cleveland, D. W. (1987) J. Cell Biol. 104, 381-383.

Cuatrecasas, P. (1970) J. Biol. Chem. 245, 3059-3065.

Dustin, P. (1984) Microtubules, Springer-Verlag, New York.

Flavin, M., Kobayashi, T., \& Martensen, T. M. (1982) Methods Cell Biol. 24, 257-263.

Gard, D. L., \& Kirschner, M. W. (1985) J. Cell Biol. 100, 764-774.

Gu, W., Lewis, S. A., \& Cowan, N. J. (1988) J. Cell Biol. 106, 2011-2022.

Job, D., Rauch, C. T., Fischer, E. H., \& Margolis, R. L. (1982) Biochemistry 21, 509-515.

Job, D., Pabion, M., \& Margolis, R. L. (1985) J. Cell Biol. $101,1680-1689$.
Job, D., Rauch, C. T., \& Margolis, R. L. (1987) Biochem. Biophys. Res. Commun. 148, 429-434.

Khawaja, S., Gundersen, G. G., \& Bulinski, J. C. (1988) J. Cell Biol. 106, 141-149.

Kirschner, M. W., \& Mitchison, T. J. (1986) Cell 45, 329-342.

Kreis, T. E. (1987) EMBO J. 6, 2597-2606.

Kumar, N., \& Flavin, M. (1982) Eur. J. Biochem. 128, 215-222.

Lewis, S. A., \& Cowan, N. J. (1988) J. Cell Biol. 106, 2023-2033.

Margolis, R. L., Rauch, C. T., \& Job, D. (1986) Proc. Natl. Acad. Sci. U.S.A. 83, 639-643.

McKinney, M. M., \& Parkinson, A. (1987) J. Immunol. Methods 96, 271-278.

Mitchison, T. J., \& Kirschner, M. W. (1984) Nature (London) 312, 237-242.

Penefsky, H. S. (1977) J. Biol. Chem. 252, 2891-2899.

Piperno, G., \& Fuller, M. T. (1985) J. Cell Biol. 101, 2085-2094.

Pirollet, F., Job, D., Margolis, R. L., \& Garel, J. R. (1987) EMBO J. 6, 3247-3252.

Sandoval, I. V., \& Cuatrecasas, P. (1976) Biochemistry 15, 3424-3432.

Schroder, H. C., Wehland, J., \& Weber, K. (1985) J. Cell Biol. 100, 276-281.

Schulze, E., Asai, D. J., Bulinski, J. C., \& Kirschner, M. W. (1987) J. Cell Biol. 105, 2167-2177.

Seitz-Tutter, D., Langford, G. M., \& Weiss, D. G. (1988) Exp. Cell Res. 178, 504-512.

Thompson, W. C. (1982) Methods Cell Biol. 24, 235-255.

Towbin, H., Staehelin, T., \& Gordon, J. (1979) Proc. Natl. Acad. Sci. U.S.A. 76, 4350-4354.

Villasante, A., Wang, D., Dobner, P., Dolph, P., Lewis, S. A., \& Cowan, N. J. (1986) Mol. Cell. Biol. 6, 2409-2419.

Wandosell, F., Serrano, L., Hernandez, M. A., \& Avila, J. (1986) J. Biol. Chem. 261, 10332-10339.

Wehland, J., \& Weber, K. (1987a) J. Cell Sci. 88, 185-203.

Wehland, J., \& Weber, K. (1987b) J. Cell Biol. 104, 1059-1067.

Wehland, J., Willingham, M. C., \& Sandoval, I. V. (1983) J. Cell Biol. 97, 1467-1475.

Wehland, J., Schroder, H. C., \& Weber, K. (1986) Methods Enzymol. 134, 171-176.

Weingarten, M. C., Lockwood, A. H., Hwo, S. Y., \& Kirschner, M. W. (1975) Proc. Natl. Acad. Sci. U.S.A. 72, 1858-1862. 